\title{
New Techniques for Non-Linear Behavioral Modeling of Microwave/RF ICs from Simulation and Nonlinear Microwave Measurements
}

\author{
David E. Root \\ Agilent Technologies \\ 1400 Fountaingrove Parkway \\ Santa Rosa, CA USA 95409 \\ 707.577.4091 \\ David_root@agilent.com
}

\author{
John Wood \\ Agilent Technologies \\ 1400 Fountaingrove Parkway \\ Santa Rosa, CA USA 95409 \\ 707.577.4702 \\ John_wood@agilent.com
}

\author{
Nick Tufillaro \\ Agilent Laboratories \\ 3500 Deer Creek Rd \\ Palo Alt, CA 94304 \\ 650.485 .8696 \\ Nick_tufillaro@agilent.com
}

\begin{abstract}
This paper compares and contrasts recent nonlinear behavioral modeling techniques designed for microwave and RFIC applications which arise in radio and communication systems, and in the design of broad-band nonlinear components used for microwave instrumentation. These techniques include dynamic neural networks and nonlinear time series models in the timedomain, nonlinear describing functions in the frequency domain, and envelope-based methods in mixed time and frequency domains. Approaches to generating these models from both simulation and nonlinear microwave measurements are reviewed.
\end{abstract}

\section{Categories and Subject Descriptors}

I.6.5 [Model Development]: Modeling methodologies

\section{General Terms}

Algorithms, Measurement, Design, Theory, Verification.

\section{Keywords}

Nonlinear Modeling, Behavioral Modeling, Nonlinear Simulation, Circuit Simulation, Nonlinear Dynamics, MMICs, RFICs
Permission to make digital or hard copies of all or part of this work for personal or classroom use is granted without fee provided that copies are not made or distributed for profit or commercial advantage and that copies bear this notice and the full citation on the first page. To copy otherwise, or republish, to post on servers or to redistribute to lists, requires prior specific permission and/or a fee.

DAC 2003, June 2-6, 2003, Anaheim, California, USA.

Copyright 2003 ACM 1-58113-688-9/03/0006 ...\$5.00

\section{INTRODUCTION}

Over the past two decades, powerful nonlinear simulation techniques have been developed to enable the efficient numerical solution of problems important to Microwave and RF ICs. Key advances have been the development of modern harmonic balance simulators, and more recently in the development of transient envelope simulators. Harmonic balance [1] allows the efficient simulation of large-signal steady-state circuits in the frequency domain. Great efficiency gains over traditional time-domain simulators like SPICE can be achieved for steady-state problems with vastly different time scales. Such problems naturally occur for circuits containing both slow and fast dynamics, such as the electro-thermal effects of RF transistors. More recently, transient envelope simulators $[2,3]$ have become available which allow the efficient simulation of problems for which the typical spectra can be represented by a set of several discrete tones and timedependent modulation around them. This is a common characteristic of modern communication circuits. Time-domain simulators, such as SPICE, have been extended to handle efficiently the steady-state solution of microwave circuits by such techniques as the shooting method and its variations [4,5]. It has been only comparatively recently, however, that commensurate nonlinear modeling techniques have arisen to make better use of these enhanced simulation capabilities.

\section{BEHAVIORAL MODELING}

A typical design and modeling hierarchy is depicted in Figure 1. At the bottom is the device. At the top is the complicated module or sub-system. A tops-down design methodology propagates specifications down the hierarchy. Bottoms up verification is the process of validating overall system performance based on the performance of lower level components and their configuration.

Behavioral models are simplified models of the essential nonlinear behavior of the components at a given level of the hierarchy for use in simulation at the next highest level of abstraction. The simplification means the models will execute more quickly, and use much less memory than if an entire complex subsystem was simulated at the transistor level. In many cases it is a practical impossibility to simulate entire modules or subsystems at the transistor level with signals characteristics of 


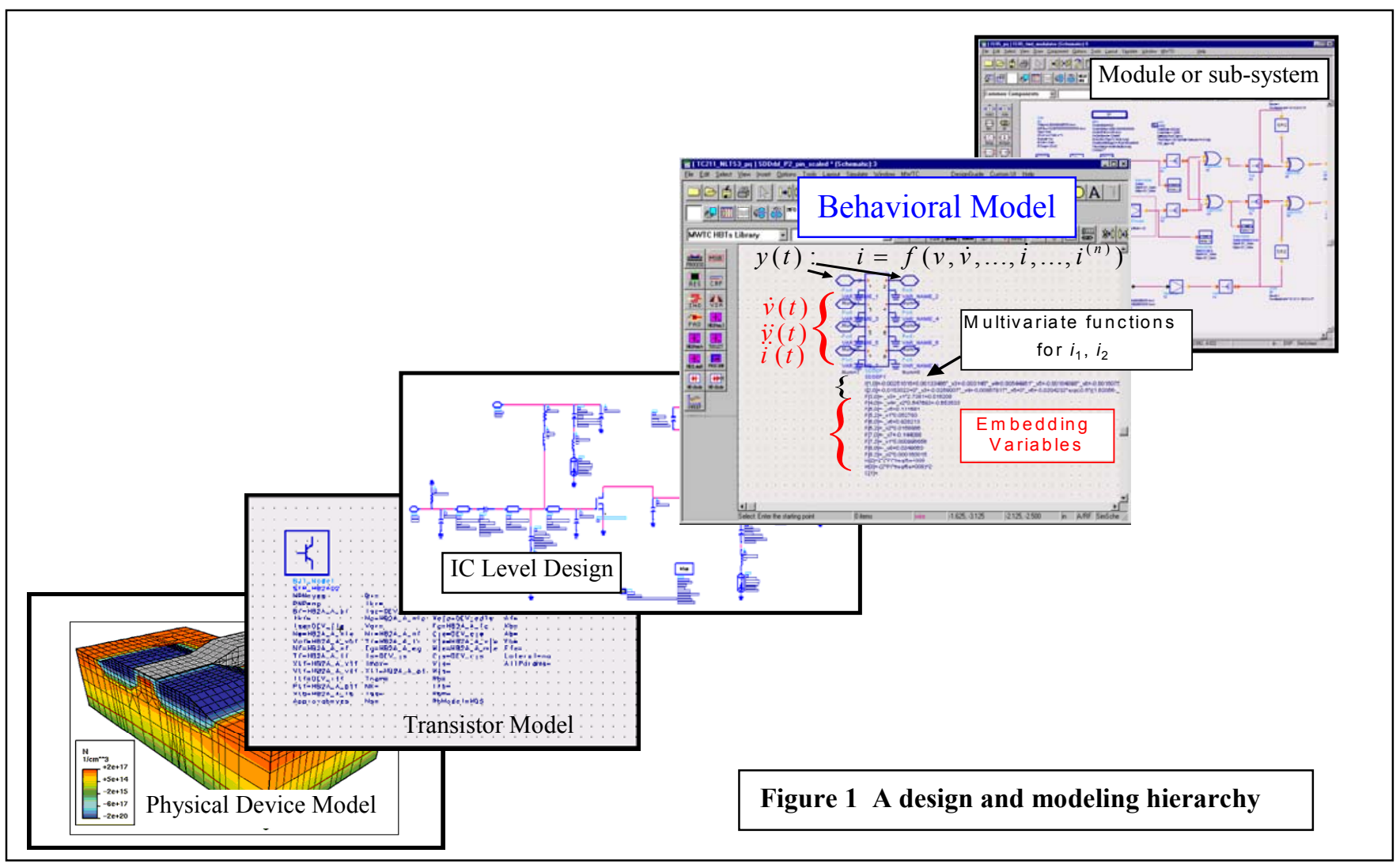

communication systems. The ability to simulate hierarchically enables optimum system design by providing bi-directional links between adjacent levels of the hierarchy. For this review, we place the behavioral model between the IC model and the module or subsystem. Many of the reviewed techniques, however, are applicable to other levels as well.

\subsection{Simulation and Measurement-Based}

Behavioral models should be derivable from both simulations and measurements. Consider a circuit model for an integrated circuit, such as that which a designer develops from primitive components such as transistors, resistors, capacitors and inductors. The simulator can be used to stimulate the IC model and the responses used to derive a behavioral model for simulation at the next higher level of abstraction. However, any modeling errors at the circuit level will be reflected in the behavioral model. That is, lower level modeling errors can accumulate from level to level up the hierarchy, invalidating the bottoms up verification.

Alternatively, behavioral models can be derived from real measurements made on the component. Accurate measurements can therefore lead to more accurate behavioral models than starting from lower level, possibly inaccurate models. This also allows designers to make behavioral models from real components for which a circuit model may not be available.

A drawback of the measurement-based approach is the lack of readily available high-frequency, nonlinear measurement systems with the required capability.
The measurement-based approach requires that the behavioral model be derivable from data that can be measured at the ports of the component connected to the external world. The same methodology can, of course, be applied in the simulator to an existing lower level model, if available.

We will only consider techniques in this review that can be applied to relationships at the external ports. This is an important restriction on the simulation-based approaches. It eliminates from consideration all order-reduction techniques based on simplifying explicit model equations and their topological connections [6].

\subsection{Desirable Properties}

Other desirable properties of behavioral models include transportability and cascadability. Transportability means the model can be used under a variety of different conditions, whether this refers to the signals stimulating the model or the environments in which the behavioral model is to be placed in the intended application. Cascadability means that the cascade of two behavioral models performs faithfully with respect to the performance of the cascade of the respective components. Since cascading nonlinear components can create a wide variety of environments for the individual behavioral models, cascadability implies a certain degree of transportability.

\subsection{Waveforms and Memory}

Behind all of the techniques described here is the notion that the behavioral model relates waveforms to waveforms. That is, the output is not an instantaneous function only of the input signal. Rather, the output depends upon the shape of the input waveform, or equivalently, the output depends on the value of the input and past values of the input, and even past values of the output itself. 
We write this in functional notation according to Equation 1.1. Here we assume the voltage signal is the input and the current is the output.

$$
I(t)=F[V(t)]
$$

The functional can often be represented as a function of the input waveform and other transforms of the input waveform. For example, we may have the relationship given by Equation 1.2.

$$
I(t)=F[V(t)]=f(V(t), \dot{V}(t))
$$

This is an example of a static functional. The output is still an instantaneous function of the input waveform, but it does not depend only on the values of the input signal, $V(t)$. A more complicated class of models is given by the feedback model of Equation 1.3.

$$
I(t)=f(V(t), \dot{V}(t), \dot{I}(t))
$$

Feedback models embody the notion of memory, or internal state. Static models such as Equation 1.2 will produce AM to PM distortion, but there is no notion of state or true memory in such a model. It is the incorporation of long-term memory beyond otherwise static nonlinear behavioral models that distinguishes the newer behavioral modeling approaches.

\subsection{Excitations}

Excitation design refers to the set of stimuli needed to elicit the full dynamics of the system response in order to identify the behavioral models. It is important to make sure the excitation is sufficiently rich to construct a behavioral model that can accurately represent signals "close" to those used in the characterization or identification. Models identified from simple excitations may be less transportable than those constructed from a varied set of excitations. This is especially true for systems with memory.

\section{TIME DOMAIN TECHNIQUES}

\subsection{Dynamic Neural Networks}

Artificial Neural Networks (ANNs) [7,8] represent nonlinear multivariate function fitting methods patterned after the nervous system. The basis set consists of nested sigmoidal functions which can universally and uniformly approximate differentiable functions in a compact subset of n-dimensional Euclidian space. Until very recently, ANNs for high-frequency electronic modeling were applied almost exclusively to static functions of the input variables alone. The application of ANNs to functionals of the inputs, that is, to functions of the input variables and their time derivatives, was first considered in [9]. This is called the method of dynamic neural networks (DNNs).

Excellent results have been obtained from the DNN method for $\mathrm{rf}$ amplifiers and mixers. Simple excitations have been used to identify these models, such as continuous wave (CW) tones swept in amplitude and frequency. In fact, simple single-tone excitations were shown to predict the intermodulation response of these circuits to two tone excitations.

DNN models are nonlinear ordinary differential equations which can therefore be implemented in generic commercial time-domain simulators. The solution of the circuit equations can be obtained using any of the simulator algorithms including harmonic balance, transient envelope, and shooting methods in the time domain.
Training, which is the process of determining the weights (or coefficients) in the basis set expansion for the ANNs, has been done in the time domain and in the frequency domain. Combined time and frequency domain training has also been reported.

Proprietary software implements the training and the attachment of the neural network model to a commercial simulator (Agilent $A D S)$.

Limitations of the DNN approach include the ad hoc nature of selecting the number of independent variables. That is, the number of time derivatives of the candidate independent variables to include as dependent variables is not an algorithmic procedure. The DNN method depends on standard issues related to neural network function fitting, including the neural net topology (number of hidden layers), number and types of neurons, and the potential issues associated with overtraining.

\subsection{Nonlinear Time Series}

Nonlienar time series (NLTS) [10-14] is a fundamentally nonlinear method based on concepts and procedures from nonlinear dynamics. The theory is routed in differential geometry and is related to the Whitney embedding theorem of manifolds $[14,15]$. The method assumes that the unknown nonlinear component of arbitrary complexity can be represented by a dynamical system, governed by state equations in canonical form as shown in Equations 1.4 and 1.5.

$$
\begin{aligned}
\dot{\vec{x}}(t) & =\vec{g}(\vec{x}(t), \vec{u}(t)) \\
y(t) & =h(\vec{x}(t), \vec{u}(t))
\end{aligned}
$$

Here $\vec{x}(t)$ is the vector of state variables, $\vec{u}(t)$ is the vector of measured inputs, and $y(t)$ is a generic measured output sampled in time (time series). The operational methodology of applying nonlinear time series is given in Figure 2.

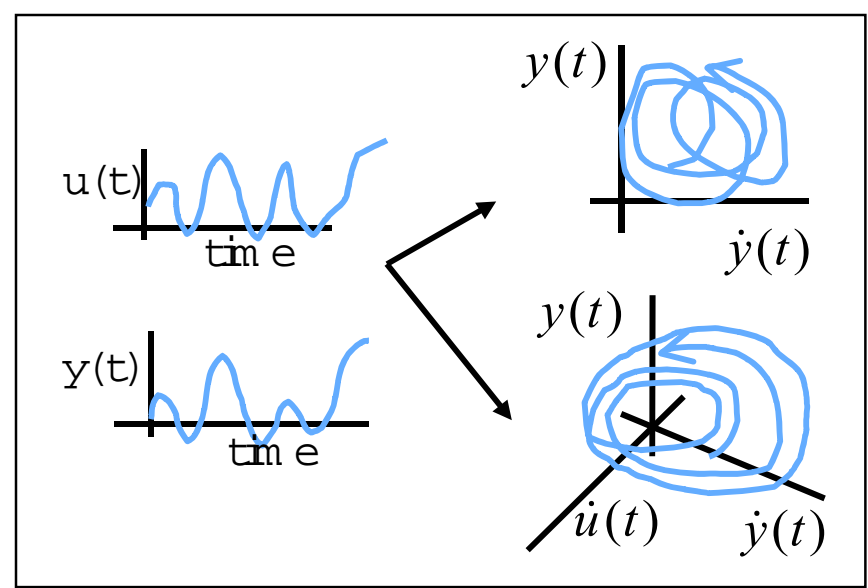

Figure 2 Non-Linear Time Series embedding process

The outputs, the inputs, and their time derivatives are unfolded in higher and higher dimensional spaces until the output becomes a single-valued function of these variables. Algorithms to find the proper choice of variables (the embedding) are based on information-theoretic principles [16]. When the trajectory becomes a single valued function, the output can then be described according to Equation 1.6. 


$$
y(t)=f\left(u(t), \dot{u}(t), \ldots, u^{(m)}(t), \ldots, \dot{y}(t), \ddot{y}(t), \ldots, y^{(n)}(t)\right)
$$

Notice that Equation 1.6 is a feedback model of the type represented by Equation 1.3. NLTS models therefore can handle systems with memory. Equation 1.6 essentially defines, implicitly, a nonlinear differential equation for the behavioral model. The function $\mathrm{f}$ is defined by suitable multivariate approximation methods. Multivariate polynomials, radial basis functions, and artificial neural networks have been used. ANNs generally give the best results, due to their asymptotic properties and because they give very smooth results for approximating discrete measured and simulated data. In [10] and [11], MATLAB was used to train the ANN functions which were then attached to the Agilent ADS simulator by custom code.

NLTS models have been constructed from simulated data and also from state-of-the-art nonlinear measurement systems of the vector nonlinear network analyzer (VNNA) type [17].

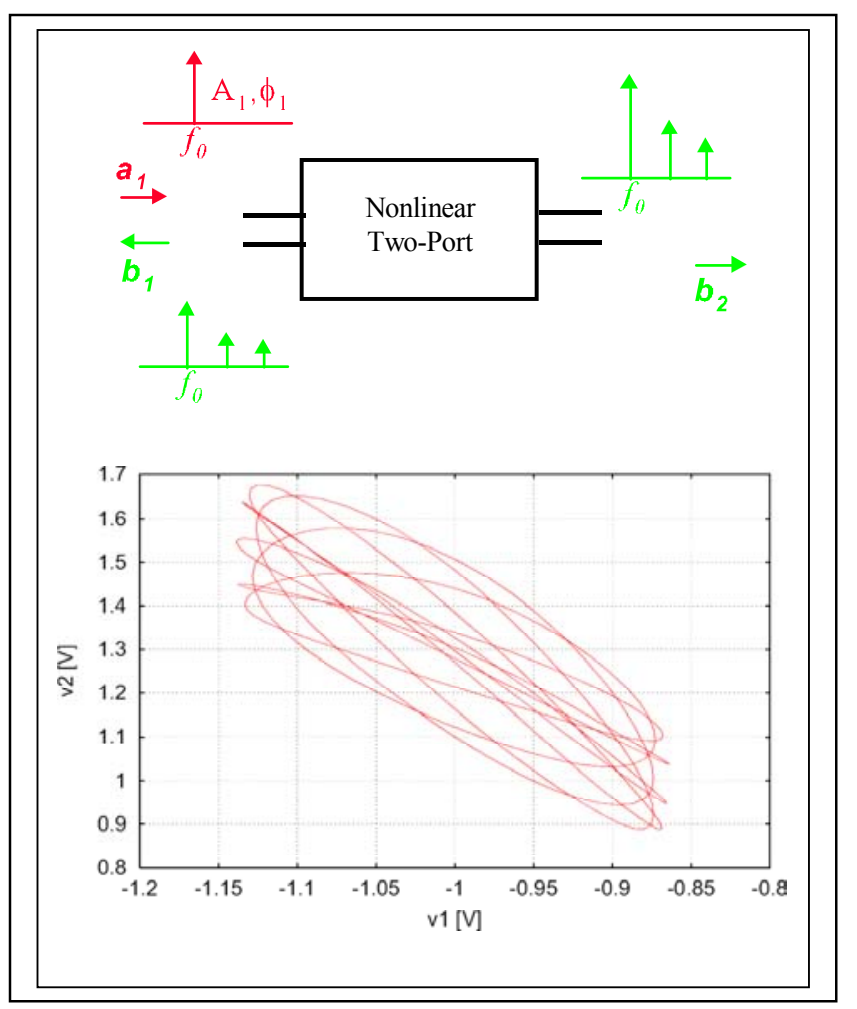

Figure 3 Nonlinear Network measurements: (a) incident waves at a single frequency scatter fundamental and harmonic frequencies. (b) by applying two offset tones at ports $1 \& 2$ a large volume of the device signal space can be covered in a single measurement.

\subsection{Comparison between methods}

The NLTS methodology can be considered a superset of the DNN approach. Up to this point, the work on DNNs has been limited to static models [9], although there is nothing fundamental about this restriction. Moreover, NLTS has associated algorithms with which to define, more systematically, the choice of variables (how many time derivatives of the variables to use for the embedding). The notion of phase space density, inherent in the nonlinear time series approach, is a way to gauge the completeness of the excitations with implications for the transportability of the model. While systems with long-term memory can be modeled with Equation 1.6, the process of NLTS model identification can get cumbersome if the problems considered have time-scales of very different orders of magnitude. A new technique is proposed to ameliorate this difficulty in [18].

\section{FREQUENCY DOMAIN TECHNIQUES}

Traditionally, microwave modeling techniques in the frequency domain have been limited to narrow-band models, that are described as memoryless or quasi-memoryless (high frequency or short-term memory). These terms correspond to the narrow-band $\mathrm{AM}$ to $\mathrm{AM}$ and $\mathrm{AM}$ to $\mathrm{PM}$ characteristics of the system, respectively. Such modeling methods have been applied to wireless power amplifiers. Excitation designs are usually simple single-tone measurements of the amplifier, and the model is identified by fitting odd-order polynomial functions to these characteristics.

Several extensions to such models to account for 'long-term' or low-frequency memory effects have been proposed in $[19,20]$ based on Wiener-Volterra theory, though mostly these approaches remain limited to narrow-band systems and weak nonlinearities.

An extension to the Volterra technique [21] allows the Volterra kernels in the nonlinear frequency domain solution to be calculated as a function of the instantaneous envelope amplitude. The kernel functions themselves are calculated from two-tone measurements using a single fixed carrier tone and a second swept tone to describe the kernel as a function of the distance in frequency from the carrier. Good results for power amplifier modeling across a multi-channel bandwidth have been demonstrated.

An attempt to incorporate this nonlinear modeling into a transient simulator has been demonstrated in [22]. Here the amplifier model itself computes the narrow-band spectral response to the time-domain modulated input. This approach is successful for a single component instance, but may be difficult to scale to multicomponent system simulation, where it would be more appropriate to perform the simulation in a harmonic balance simulator

\subsection{Describing Functions}

The describing function technique is an inherently nonlinear formulation based in the frequency domain [23]. The model is based on the assumption of a harmonic balance solution of the circuit equations, which can therefore be written as Fourier Series according to Equations 1.7 and 1.8. The expansion coefficients are complex number representing the harmonic components of the signal.

$$
\begin{gathered}
V(t)=\operatorname{Re} \sum_{k} V_{k} \exp \left(j \omega_{k} t\right) \\
I(t)=\operatorname{Re} \sum_{k} I_{k} \exp \left(j \omega_{k} t\right)
\end{gathered}
$$

A describing function model can be defined by the complex mapping relating the $I_{k} \mathrm{~s}$ as nonlinear functions of the $V_{k} \mathrm{~s}$. Usually, however, a change of variables is done and the describing 
functions are defined in terms of incident and scattered waves rather than voltages and currents. The complex scattered wave is therefore represented as a nonlinear complex (but not analytic) function of the incident waves given by Equation 1.9.

$$
\vec{b}=\vec{f}(\vec{a})
$$

The components of the vector represent the harmonic components of the waves in the frequency domain. The length of the vector corresponds to the number of frequency components kept in the approximation of the signal. In this theory, the waves have an absolute size associated with them, unlike the case of (linear) Sparameters which are defined only in terms of ratios of scattered to incident waves. Describing functions reduce to standard Sparameters in the limit of small signals.

The function $\mathrm{f}$ in Equation 1.9 is expanded in a suitable bases set and fitted from a set of data obtained by measuring (or simulating) the response of the systems to incident waves of varying amplitudes and recording the magnitude and phase of the response. Applications based on real microwave measurements require the VNNA instrument described earlier, in order to get the relative phases between successive harmonics. The model can be directly implemented in a harmonic balance simulator by using, for example, a frequency domain nonlinear modeling element, such as the Frequency Domain Device (FDD) in Agilent ADS.

A useful intermediate case exists between the full nonlinear describing functions and the linear S-parameter case. In [24] the describing functions are linearized around the fundamental response to a large amplitude signal, which can be highly nonlinear. The resulting approximate model has the property that it is linear in the harmonics around this fundamental response. This "harmonic superposition" approximation is useful in simplifying the excitation design and the subsequent identification of the approximate model for a power amplifier.

Unlike DNN and NLTS models, which can be used in all modes of simulation, describing function models can be used only in harmonic balance and linear analysis in the frequency domain. They can not be used directly to simulate the response of a system to modulated signals such as W-CDMA.

\section{ENVELOPE METHODS}

These techniques combine some aspects of the time domain formulations with some aspects of the frequency domain techniques. The basic assumption is that the solution of the circuit equations can be represented by a Fourier Series, similar to Equations 1.7 and 1.8, but this time the coefficients of this expansion can vary in time. That is, the solution of the circuit equations is assumed to be given by equations 1.10 and 1.11:

$$
\begin{gathered}
V(t)=\operatorname{Re} \sum_{k} V_{k}(t) \exp \left(j \omega_{k} t\right) \\
I(t)=\operatorname{Re} \sum_{k} I_{k}(t) \exp \left(j \omega_{k} t\right)
\end{gathered}
$$

These time-dependent coefficients are the complex envelopes. The transient envelope solution technique $[2,3]$ is efficient provided that the time varying coefficients vary slowly compared with the carriers. This is usually the case for narrow-band communication circuits excited by digitally modulated signals.
By addressing the envelope behavior in the time-domain, the long-term memory effects can be included in the nonlinear model. Systems with long-term memory can have very different behaviors depending on the dynamics of the envelopes It becomes important to stimulate the system in ways that affect the dynamics of the envelopes. Excitation designs will need to go well beyond the conventional two-tone techniques. Using digitally modulated signals or band-limited noise may be necessary. Specialized hardware systems have been developed to do this [17,21].

A key requirement is the ability for the modeler to interface with the envelope simulator. Some models are based on variables characteristic of the envelope and RF carriers independently. Most simulators do not yet have the interface to directly enable modelers to exploit such behavioral models.

\section{CONCLUSIONS}

Behavioral modeling techniques have recently been increasing in sophistication and capability. Several techniques, notably those in the frequency domain and the envelope domain, are intimately related to the underlying simulator algorithm used in their solution. Successful application of behavioral modeling techniques to important design challenges will depend upon the ease of interfacing with the underlying simulation engine, the ability to systematically apply the techniques to significant quantities of data, and the ability to distribute these behavioral models to customers. Finally, the ability to perform waveform measurements at microwave frequencies in order to apply these techniques to real data will be a big advantage to get around accuracy limitations of building behavioral models from lower level models.

\section{ACKNOWLEDGMENTS}

Thanks to Hassan Tanbakuchi, Dominique Schreurs, Alex Pekker, Darryl Okahata, Alex Cognata, Brian Hughes, Derry Hornbuckle, Jerry Gladstone, and Agilent Technologies Management for supporting this work.

\section{REFERENCES}

[1] Kundert, K. S., Sangiovanni-Vincentelli. A., Simulation of nonlinear circuits in the frequency domain. IEEE Transactions on Computer-Aided Design of Integrated Circuits and Systems CAD-5(4) pp.521-535 (1986).

[2] Sharrit, D. A new method of analysis of communication systems. IEEE MTTS'96 Workshop on Nonlinear CAD (June 1996).

[3] Ngoya, E, and Larcheveque, R. Envelop transient analysis: a new method for the transient and steady state analysis of microwave communication circuits and systems. IEEE MTTS'96 (June 1996) pp1365-68.

[4] Kundert, K. S., White, J. K., Sangiovanni-Vincentelli, A. An envelope-following method for the efficient transient simulation of switching power and filter circuits. IEEE ICCAD-88 International Conference on Computer-Aided Design (November 1988) pp. 446-449.

[5] Kundert, K. S., White, J. K., Sangiovanni-Vincentelli, A. Steady-State Methods for simulating analog and microwave circuits. Kluwer Academic Publishers 1990 
[6] Phillips, J.R. Projection-based approaches for model reduction of weakly nonlinear time-varying sytesms. IEEE Trans. Computer-aided design of integrated circuits and systems, Vol 22, No. 2, (February, 2003)

[7] Zhang, Q. J. and Gupta, K. C. Neural networks for RF and microwave design. Artech House (2000).

[8] Haykin, S. Neural Networks: a comprehensive foundation. 2nd ed. Prentice-Hall (1999).

[9] Xu, J., Yagoub, M. C. E., Ding, R., Zhang, Q. J. Neural based dynamic modeling of nonlinear microwave circuits. IEEE MTT-S 2002 (June 2002) pp. 1101-1104.

[10] Wood, J. and Root, D. E. The behavioral modeling of microwave/RF ICs using non-linear time series analysis. IEEE IMS2003 (June 2003).

[11]Root, D. E., Wood, J., Pekker, A. Systematic behavioral modeling of nonlinear microwave/RF circuits in the time domain using techniques from nonlinear dynamical systems. IEEE BMAS 2002 (October 2002).

[12] Schreurs, D., Wood, J., Tufillaro, N., Barford, L. and Root, D. E. Construction of behavioural models for microwave devices from time-domain large-signal measurements to speed up high-level design simulations. International Journal of RF and Microwave Computer-Aided Engineering: Special Issue on 'Measurement Techniques for Effective CAD' 13(1) pp. 54-61 (2003).

[13] Schreurs, D.,Wood, J., Tufillaro, N., Usikov, D., Barford, L. and Root, D. E. The construction and evaluation of behavioral models for microwave devices based on timedomain large-signal measurements. International Electron Devices Meeting 2000, (December 2000) IEDM Technical Digest pp. 819 -822.

[14] Kantz, H. and Schreiber, T. Nonlinear Time Series Analysis, Cambridge University Press (1997)

[15] Stark, J. Delay embeddings and forced systems, J. Nonlinear Science, 9 pp255-332 (1999).
[16] Kennel, M. B., Brown, R., Abarbanel., H. Determining embedding dimension for phase-space reconstruction using a geometrical construction, Phys. Rev. A 45 pp3403-3411 (1992).

[17] Verspecht, J., Verbeyst, F.. Vanden Bossche, M. Network analysis beyond S-parameters: characterizing and modeling component behaviour under modulated large-signal operating conditions. ARFTG 56 Colorado (December 2000)

[18] Schreurs, D. and Remley, K. Use of multisine signals for efficient behavioural modelling of RF circuits with shortmemory effects. ARFTG 61Philadelphia (June 2003).

[19] Ku, H., McKinley, M. D., Kenney, J. S. Extraction of accurate behavioral models for power amplifiers with memory effects using two-tone measurements. IEEE MTT-S 2002 (June 2002) pp. 139-142.

[20]Reig, P., LeGallou, N., Nebus, J-M., and Ngoya, E. Accurate RF and microwave system level modeling of wideband nonlinear circuits. IEEE MTT-S2000 (June 2000) pp. $79-82$.

[21] LeGallou, N., Ngoya, E., Buret, H., Barataud, D., and Nebus, J-M. An improved behavioral modeling technique for high power amplifiers with memory. IEEE MTT-S2001 (May 2001) pp. 983-986.

[22] Tuinenga, P., Models rush in where simulators fear to tread: extending the baseband-equivalent method. . IEEE BMAS 2002 (October 2002).

[23] Verspecht, J. Describing functions can better model hard nonlinearities in the frequency domain than the Volterra theory. Annex to Ph.D. dissertation, Vrije Universiteit Brussel (1995).

[24] Verspecht, J., Verbeyst, F.. Vanden Bossche, M., Van Esch, P. System level simulation benefits from frequency domain behavioral models of mixers and amplifiers, in Proceedings of 29th European Microwave Conf. (1999)., pp29-32. 